\title{
Wood-inspired engineering materials
}

\section{Lennart Bergström}

Nature continues to serve as inspiration for the design and fabrication of high-performance materials [1,2]. The structural and compositional features responsible for e.g. the high hardness and fracture toughness of nacre, tooth enamel and other biominerals have served as a benchmark for the structural design of hard and strong materials [3]. Wood has been utilized by humankind for millennia for protection, construction, energy and for cultural and religious objects. The hierarchical and cellular structure of wood and the structure-property relations of the constituents and their assemblies: e.g. cellulose, hemicellulose and lignin, has been the focus of intense research efforts and resulted in advances in the fabrication of wood-based functional and structural highperformance materials $[4,5]$.

Recently, a team led by Shu-Hong Yu from the University of Science and Technology of China (USTC) developed a family of synthetic polymeric woods through an "assembly-and-thermocuring" strategy using traditional phenolic resin and melamine resin as the matrix materials (Fig. 1a-d) [6]. The liquid thermoset resins were unidirectionally frozen and freeze dried to generate a cellular structure with aligned pores. The polymer chitosan maintains the hierarchical architecture during processing and subsequent thermocuring. The artificial woods bear a close resemblance to natural woods in the mesoscale cellular structures with tunable pore size and wall thickness (Fig. 1e).

The artificial woods manifest compressive strengths
( $45 \mathrm{MPa})$ comparable to that of the natural woods and higher than that of many engineering materials with similar densities (Fig. 1f). The use of synthetic resins and a variety of inorganic and organic fillers in the artificial woods creates a versatile cellular material with significantly better moisture and fire resistance compared to natural woods. The biomimetic strategy demonstrated by Yu et al. could open up the design of materials suitable for e.g. packaging and thermal insulation. The interest in the materials would certainly increase if the synthetic resins can be replaced by bio-based substitutes, and if scalable alternatives to the time- and energy-demanding freeze casting and freeze drying process could be developed.

\section{Received 11 August 2018; accepted 11 August 2018;} published online 22 August 2018

1 Meyers MA, McKittrick J, Chen PY. Structural biological materials: critical mechanics-materials connections. Science, 2013, 339: 773779

2 Fratzl P, Barth FG. Biomaterial systems for mechanosensing and actuation. Nature, 2009, 462: 442-448

3 Espinosa HD, Soler-Crespo R. Lessons from tooth enamel. Nature, 2017, 543: 42-43

4 Song J, Chen C, Zhu S, et al. Processing bulk natural wood into a high-performance structural material. Nature, 2018, 554: 224-228

5 Wicklein B, Kocjan A, Salazar-Alvarez G, et al. Thermally insulating and fire-retardant lightweight anisotropic foams based on nanocellulose and graphene oxide. Nat Nanotech, 2015, 10: 277283

6 Yu ZL, Yang N, Zhou LC, et al. Bioinspired polymeric woods. Sci Adv, 2018, 4: eaat7223

Department of Materials and Environmental Chemistry, Stockholm University, Stockholm 10691, Sweden Email: lennart.bergstrom@mmk.su.se 
a

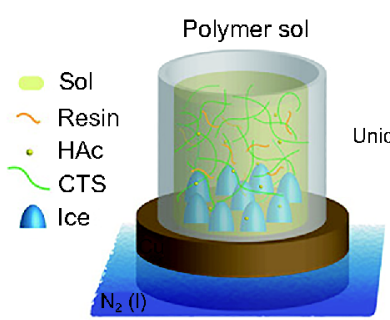

d

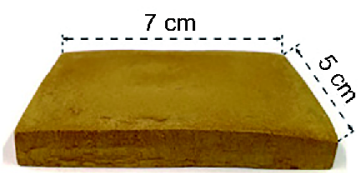

Phenolic wood

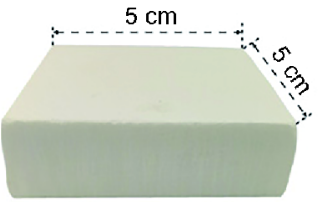

Melamine wood

e
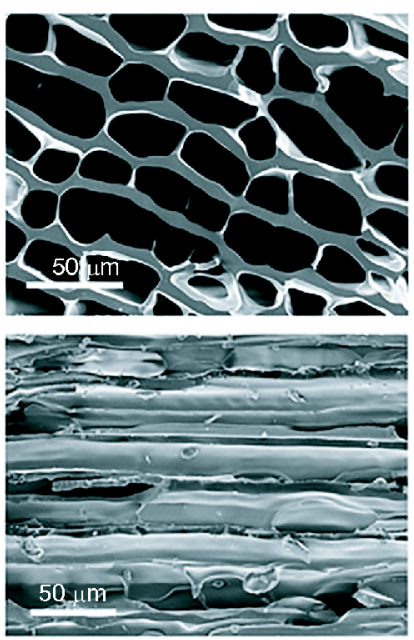

directional freezing

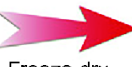

Polymer cryogel

b

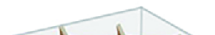

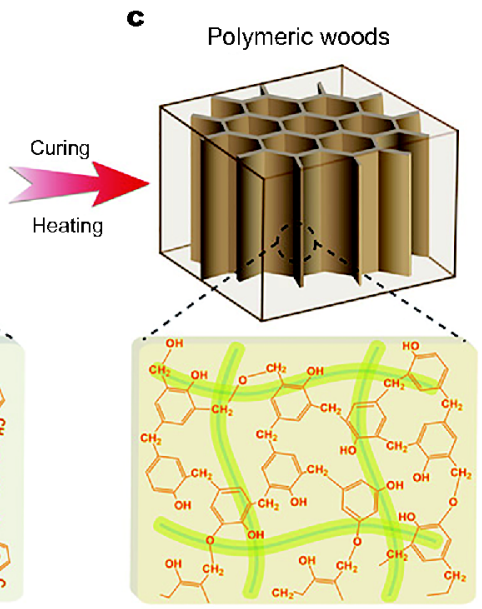

PF
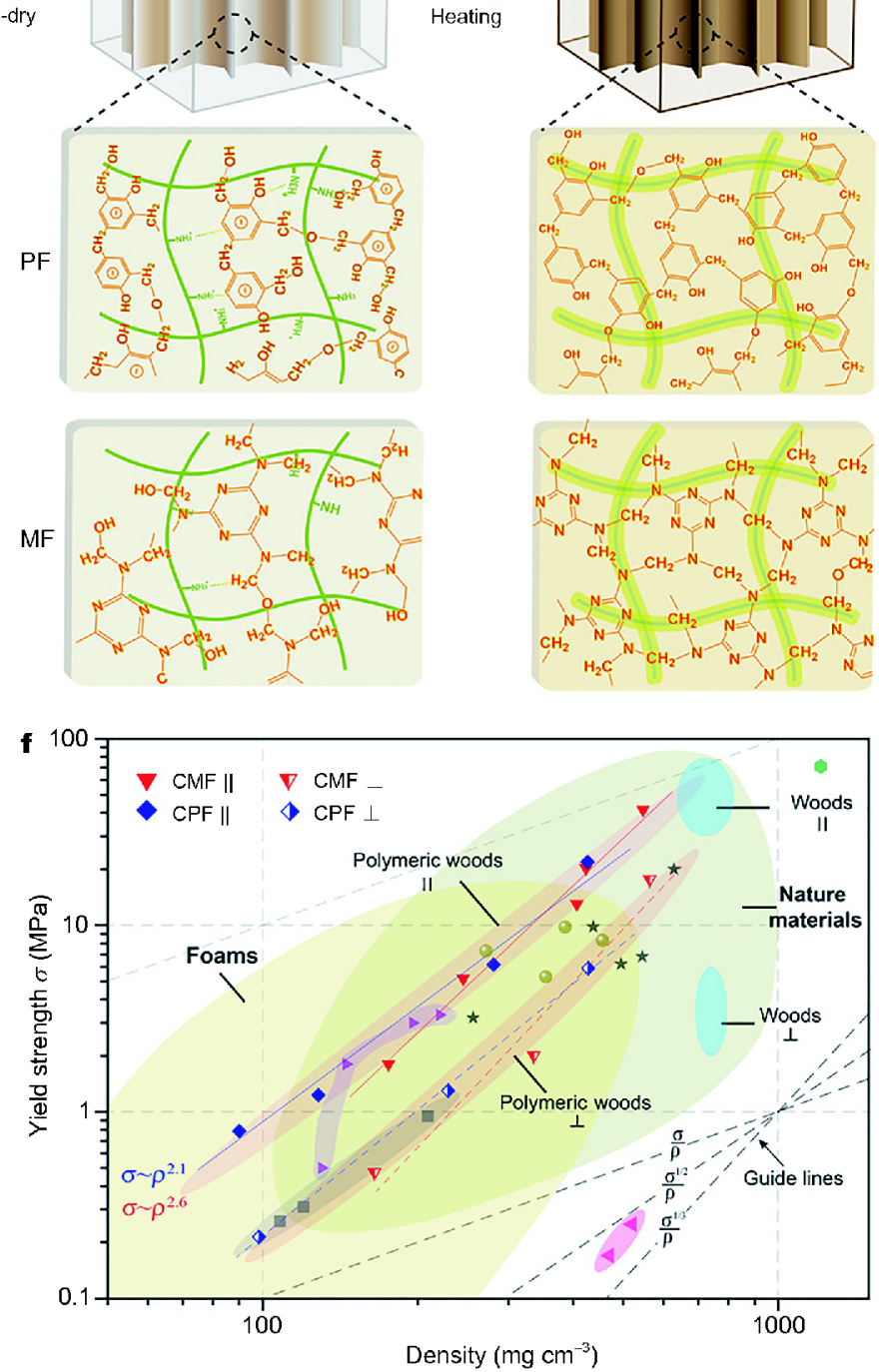

Figure 1 The bio-inspired artificial woods based on the phenolic resin and melamine resin. (a) The homogeneous polymer sol including water soluble thermoset resins and chitosan. (b) The predesigned "green body" prepared by the ice template induced self-assembly and freeze-drying process. (c) The artificial woods by thermocuring the predesigned matrix. (d) The two kinds of typical artificial woods. (e) The SEM images of the cross section and the longitudinal section of a typical phenolic wood. (f) An Ashby chart plotting compressive yield strength versus density for the artificial woods and other engineered materials. Reprinted with permission from Ref. [6], Copyright 2018, American Association for the Advancement of Science. 